\title{
EDITORIAL
}

\section{Generalized anxiety disorder: advances in neuroimaging studies}

\author{
Marianna de Abreu Costa, ${ }^{1}$ (iD Gisele Gus Manfro ${ }^{1,2}$ (iD \\ ${ }^{1}$ Programa de Pós-Graduação em Psiquiatria e Ciências do Comportamento, Faculdade de Medicina, Universidade Federal do Rio Grande do \\ Sul (UFRGS), Porto Alegre, RS, Brazil. ${ }^{2}$ Programa de Transtornos de Ansiedade, Hospital de Clínicas de Porto Alegre (HCPA), Porto Alegre, \\ RS, Brazil.
}

Anxiety disorders are the most prevalent mental disorders. ${ }^{1}$ According to the Global Burden of Disease Study, anxiety disorders are the sixth leading cause of disability worldwide, and even mild syndromes are associated with impairment and distress. ${ }^{2}$ Those affected with anxious symptoms have a lower quality of life and poorer psychosocial functioning. ${ }^{3}$ Despite the many available treatments, which include antidepressants and cognitive behavioral therapy (CBT), ${ }^{4}$ only $60 \%$ of patients improve. ${ }^{5,6}$ Those patients with residual symptoms tend to experience a waxing and waning pattern of recurrence, leading to a chronic course. Very little evidence is available to guide treatment selection (CBT, selective serotonin reuptake inhibitors [SSRIs], or a combination thereof) for the individual patient. ${ }^{7}$ Advances in neurosciences and studies designed to understand the mechanisms and biomarkers associated with these disorders may help guide personalized treatment approaches. Moreover, a better understanding of the disorders themselves, their pathological pathways, biomarkers, and clinical aspects (risk factors and predictors of response) may help develop treatments that are more effective. Selecting the optimal treatment for a specific patient would reduce individual and social costs.

In agreement with this context, the present issue of the Brazilian Journal of Psychiatry publishes a systematic review of brain imaging studies in patients with generalized anxiety disorder (GAD). ${ }^{8}$ This review provides some inspiring data, despite the methodological heterogeneity of the included studies. Noting that results could be influenced by differences in study design, variation in image acquisition and processing, and the presence of comorbidities, the authors nevertheless present consistent data of an association of decreased connectivity between prefrontal, limbic, and cingulate areas with core symptoms of GAD, such as worry severity. Moreover, they report altered connection between the amygdala and default mode and salience networks associated with cognitive and emotional processes in GAD. Some studies also evaluated neurological functions during the activation paradigm before and after GAD treatment, and reported that higher baseline reactivity to fearful faces in the rostral anterior cingulate cortex, as well as dorsolateral and ventrolateral prefrontal cortex reactivity, were associated with better treatment response. These findings suggest that neuroimaging might be used as a predictor of treatment response in the future.

Despite some limitations, these are promising preliminary findings regarding GAD pathophysiology. The neuronal basis of GAD deficits is associated with hypoactivation of the prefrontal cortex and hyperactivation of the amygdala, and may also encompass areas involved in the emotional process, especially the prefrontal-limbic network. We hope these findings, however preliminary, can guide future studies to a deeper understanding of GAD mechanisms and to the development of more effective and personalized treatment approaches.

\section{Disclosure}

The authors report no conflicts of interest.

\section{References}

1 Kessler RC, Angermeyer M, Anthony JC, DE Graaf R, Demyttenaere K, Gasquet I, et al. Lifetime prevalence and age-of-onset distributions of mental disorders in the World Health Organization's World Mental Health Survey Initiative. World Psychiatry. 2007;6: 168-76.

2 Baxter AJ, Vos T, Scott KM, Ferrari AJ, Whiteford HA. The global burden of anxiety disorders in 2010. Psychol Med. 2014;44:2363-74.

3 Mendlowicz MV, Stein MB. Quality of life in individuals with anxiety disorders. Am J Psychiatry. 2000;157:669-82.

4 Katzman MA, Bleau P, Blier P, Chokka P, Kjernisted K, Van Ameringen $M$, et al. Canadian clinical practice guidelines for the management of anxiety, posttraumatic stress and obsessive-compulsive disorders. BMC Psychiatry. 2014;14 Suppl 1: S1.

5 Bystritsky A. Treatment-resistant anxiety disorders. Mol Psychiatry. 2006;11:805-14.

6 Springer KS, Levy HC, Tolin DF. Remission in CBT for adult anxiety disorders: a meta-analysis. Clin Psychol Rev. 2018;61:1-8.

7 Craske MG, Stein MB. Anxiety. Lancet. 2016;388:3048-59.

8 Madonna D, Delvecchio G, Soares JC, Brambilla P. Structural and functional neuroimaging studies in generalized anxiety disorder: a systematic review. Braz J Psychiatry. 2019;41:336-62. 